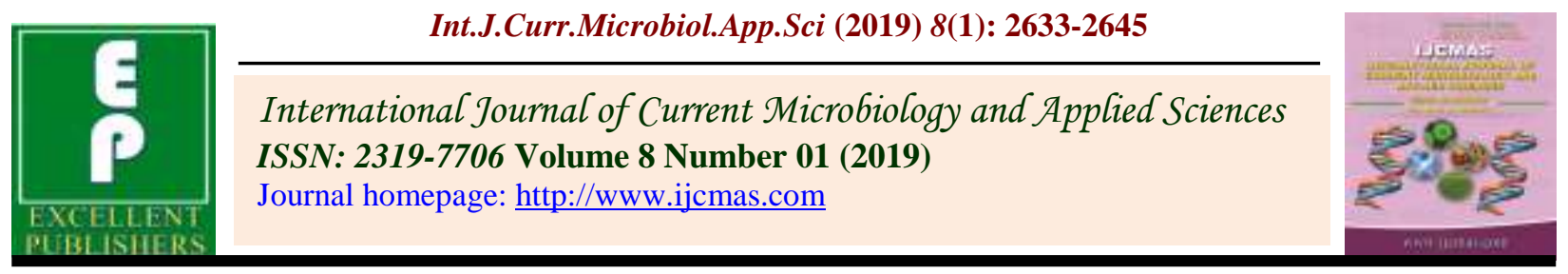

Original Research Article

https://doi.org/10.20546/ijcmas.2019.801.277

\title{
Identification of Plant Growth Promoting Rhizobacteria as Biofertilizer for Salt Stress Environment
}

\author{
Ajay Kumar Singh and Padmanabh Dwivedi* \\ Department of Plant Physiology, Institute of Agricultural Sciences, Banaras Hindu University, \\ Varanasi, India-221005, India \\ *Corresponding author
}

A B S T R A C T

Keywords

Auxin, Hydrogen cyanide, PGPR,

Phosphorus,

Potassium,

Pseudomonas

aeruginosa,

Siderophore

Article Info

Accepted:

17 December 2018

Available Online:

10 January 2019
Plant growth promoting rhizobacteria are found in the rhizosphere of plants worldwide and enhance their growth and development under unfavorable environmental condition. In the present investigation, twelve plant growth promoting rhizobacteria were isolated from the rhizosphere of different plants. Among these isolated rhizobacteria, identified one out of twelve, which showed best biofertilizer activity efficiently and also performing well under salt stress environment. Such biofertilizer activities were observed as solubilization of phosphorus and potassium, production of iron sequester complex-siderophore, production of auxin (a plant growth regulator) and production of hydrogen cyanide (an anti-microbial compound). The best performing rhizobacterial isolate, which showed best biofertilizer activity, was confirmed as Pseudomonas aeruginosa based on 16S rRNA sequencing under salt stress conditions.

\section{Introduction}

Rhizosphere is the most important ecological niche comprising different types of soil surrounding plant root zone with maximum beneficial bacterial population that are influenced by root exudates. Rhizobacterial population in the rhizospheric root zone are 100-1,000 times higher than in bulk soil and occupy metabolic flexibility to modify the soil composition efficiently by utilizing the root exudates (Jha et al., 2010; Govindasamy et al., 2011). Plant roots secreted photosynthates in the soil about 5 to $30 \%$ in form of different sugars that is utilized by microbial populations (Glick, 2014). Subsequent metabolic activities of these rhizobacteria stimulate plant growth and development by solubilising mineral nutrient present in the insoluble form in the soil (Glick, 1995). Plant Growth Promoting Rhizobacteria (PGPR) is beneficial bacteria to the plant growth under both biotic and abiotic stress environment. PGPR have ability to colonize around the root zone and encourage plants for their growth and development through either direct or indirect mechanisms. In the direct mechanisms, which can be 
correlated with their capability to produce iron chelator compound siderophore, indole acetic acid (IAA), solubilise phosphorus, potassium, exo-polysaccharide and ACC deaminase activity directly help the plant in several ways: (1) Provide nutrient availability to the plant by solubilizing unavailable or fixed nutrients in the soil (2) Enhance plant growth by producing phytohormone (3) Encourage plant growth from abiotic stresses i.e. drought, salinity and water logging through reducing the production of ethylene by ACC deaminase activity and (4) Check the entry of $\mathrm{Na}^{+}$salt into the plant cell through formation of exopolysaccharide (EPS) around the root surface. Indirectly, by releasing antifungal and anti- bacterial compounds i.e. hydrogen cyanide and ammonia that reduce the pathogenic microbial population. PGPR include bacteria that reside in the rhizosphere and improve plant health ultimately boosting up the plant growth.

Majority of PGPR belongs to genera Acinetobacter, Agrobacterium, Arthobacter, Azotobacter, Azospirillum, Burkholderia, Bradyrhizobium, Rhizobium, Frankia, Serratia, Thiobacillus, Pseudomonads and Bacillus (Vessey, 2003; Dobbelaere et al., 2003; Sheng, 2005; Han et al., 2006; Spaepen et al., 2007; Hayat et al., 2010; Mishra et al., 2010; Park et al., 2010; Tallapragada, 2010; Yousefi et al., 2011; Liu et al., 2012; Ahmed and Holmstrom, 2014; Retha et al., 2014). Other than PGPR, plant growth promoting fungi (PGPF) are also present in the soil around the root zone that produced beneficial effects in terms of plant growth promotion and biological control resulting in enhanced plant growth and development (Hoitink et al., 2006; Mathys et al., 2012; Singh et al., 2015; Singh and Dwivedi, 2018a). A specific PGPF like Trichoderma have been shown to have ameliorated the hostile effects on plants, increasing their growth potential, nutrient uptake, rate of seed germination and stimulation of plant defence against biotic and abiotic damages (Shoresh et al.,2010; Singh et al., 2015; Singh and Dwivedi, 2018a; Singh and Dwivedi, 2018b).

\section{Materials and Methods}

\section{Isolation and purification of isolated rhizobacteria}

Rhizobacteria were isolated from the rhizosphere of Solanum melongena, Capsicum annuum and Solanum lycopersicum from different districts of Uttar Pradesh (Varanasi, Mirzapur and Sonbhadra) and Uttarakhand (Pant Nagar), India. Isolated rhizobacteria were purified by dissolving $1 \mathrm{~g}$ of rhizospheric soil in $10 \mathrm{~mL}$ of distilled water. Using this as stock, $100 \mu \mathrm{l}$ of soil solution was taken from test tube containing $10^{-6}$ and $10^{-7}$ concentration in autoclaved plates of nutrient media containing $5 \mathrm{~g}$ peptone, $3 \mathrm{~g}$ beef extract, $5 \mathrm{~g}$ sodium chloride, $15 \mathrm{~g}$ agar, $1 \mathrm{~L}$ distilled water and $\mathrm{pH} 6.8 \pm 0.5$. Soil solution was spread with the help of spreader and finally incubated at temperature $28^{\circ} \mathrm{C}$ for $48-72 \mathrm{~h}$. Single bacterial colony was isolated from incubated plates after $48-72 \mathrm{~h}$ and, for purification, inoculated single bacterial colony on fresh media and then performing several biofertilizer tests with these single bacterial colonies.

\section{Phosphate solubilization efficiency}

The phosphate solubilization abilities of different isolated rhizobacteria were evaluated qualitatively according to the methods of Mehta and Nautiyal (2001).

This method is based on the decolourization of bromophenol blue (BPB) following a decrease in $\mathrm{pH}$ of the culture medium. All the rhizobacteria were inoculated separately in the plates of NBRI-BPB medium. This media containing $10 \mathrm{~g}$ of sucrose, $5 \mathrm{~g}$ of $\mathrm{Ca}_{3}\left(\mathrm{PO}_{4}\right)_{2}$, 
$5 \mathrm{~g}$ of $\mathrm{MgCl}_{2} \cdot 6 \mathrm{H}_{2} \mathrm{O}, 0.25 \mathrm{~g}$ of $\mathrm{MgSO}_{4} \cdot 7 \mathrm{H}_{2} \mathrm{O}$, $0.2 \mathrm{~g}$ of $\mathrm{KCl}, 0.1 \mathrm{~g}$ of $\left(\mathrm{NH}_{4}\right)_{2} \mathrm{SO}_{4}$ and $0.025 \mathrm{~g}$ of BPB were dissolved in $1000 \mathrm{~mL}$ of distilled water. Isolated pure cultures of rhizobacteria was inoculated on previously poured NBRI-BPB media, and transferred to the incubator at temperature $26 \pm 2^{\circ} \mathrm{C}$. Finally, data were recorded at 5, 10 and 15 days after inoculation, if blue colour on media disappeared, and calculated phosphorus solubilization efficiency (PSE) using the formula given below:

$$
\mathrm{PSE}=\frac{\text { Zone of decolourization }(\mathrm{cm})}{\text { Bacterial growth }(\mathrm{cm})} \times 100
$$

\section{Potassium solubilization efficiency}

Potassium solubilizing capabilities of purified isolated rhizobacteria from different rhizospheres of plants were evaluated qualitatively on Aleksandraov medium ( $\mathrm{Hu}$ et al., 2006) with some modifications. This media contained $5.0 \mathrm{~g}$ of Glucose, $0.005 \mathrm{~g}$ of $\mathrm{MgSO}_{4} .7 \mathrm{H}_{2} \mathrm{O}, 0.1 \mathrm{~g}$ of $\mathrm{FeCl}_{3}, 2.0 \mathrm{~g}$ of $\mathrm{CaCO}_{3}$, $3.0 \mathrm{~g}$ of potassium mineral (mica), $2.0 \mathrm{~g}$ of $\mathrm{Ca}_{3}\left(\mathrm{PO}_{4}\right)_{2}, 20.0 \mathrm{~g}$ agar and $1.0 \mathrm{~L}$ of distilled water and recorded potassium solubilization efficiency (PSE) at 5, 10 and 15 days after inoculation by using the same formula, which is given in phosphate solubilization test.

\section{Siderophore production}

Evaluation of siderophore production abilities of isolated rhizobacteria was done qualitatively by the methods of Schwyn and Neilands (1987). Freshly prepared chrome azurole $\mathrm{S}$ mixture containing $60.5 \mathrm{mg}$ of chrome azurole $S$ in $50 \mathrm{ml}$ water, $10 \mathrm{mM}$ of $\mathrm{FeCl}_{3} \cdot 6 \mathrm{H}_{2} \mathrm{O}$ in $10 \mathrm{ml}$ of $10 \mathrm{mM} \mathrm{HCl}$ and 72.9 $\mathrm{mg}$ of HDTMA in $40 \mathrm{ml}$ of distilled water were prepared separately and mixed in the ratio of 5:1:4, respectively, and the final azurole $\mathrm{S}$ mixture solution was mixed with nutrient media in 1:3 ratio. Siderophore production was observed at 5,10 and 15 days after inoculation by measuring dark red zone appeared on inoculated plates, and calculated siderophore production efficiency by using the same formula, which is given above.

\section{Auxin production}

Auxin production abilities of different isolated rhizobacteria were evaluated qualitatively by freshly prepared NATD media containing $35 \mathrm{~g}$ of nutrient agar, $0.9 \mathrm{~g}$ of tryptophane, $0.6 \mathrm{~g}$ of SDS and $10 \mathrm{~mL}$ of glycerol dissolved in $1000 \mathrm{~mL}$ of distilled water under aseptic condition, and put the sterilized filter paper over the inoculated rhizobacteria.

Then, transferred inoculated plates into BOD for overnight at $26 \pm 2{ }^{\circ} \mathrm{C}$. Removed the filter paper next day, and treated with salkowaski reagent, prepared by the methods of Ehmann (1977). If bacteria produced auxin, it formed red or pink halo within the membrane surrounding the colony. Measured the diameter of red or pink halo appeared on filter paper.

\section{Hydrogen cyanide (HCN) production}

Screening of purified rhizobacteria isolated from different plants was evaluated for $\mathrm{HCN}$ production by methods of Castric (1975). Bacterial cultures were streaked on nutrient medium containing $20 \mathrm{~g}$ of peptone, $15 \mathrm{ml}$ of glycerol, $1.5 \mathrm{~g}$ of $\mathrm{K}_{2} \mathrm{HPO}_{4}, 1.5 \mathrm{~g}$ of $\mathrm{MgSO}_{4} \cdot 7 \mathrm{H}_{2} \mathrm{O}, 20 \mathrm{~g}$ of Agar and $1 \mathrm{~L}$ of distilled water.

A Whatman filter paper No. 1 soaked in $0.5 \%$ picric acid solution (in $2 \%$ sodium carbonate) was placed inside the lid of a plate. Plates were sealed with parafilm and incubated at $26 \pm 2{ }^{\circ} \mathrm{C}$. Development of light brown to dark brown color indicated $\mathrm{HCN}$ production.

Colony forming unit (CFU $\left.\mathrm{mL}^{-1}\right)$ of 
isolated rhizobacteria under $2 \%$ and $5 \%$ NaCl solution

Finally, among isolated rhizobacterial strains, selected single rhizobacteria that showed the best PGPR activities in several tests and well survived under salty conditions (2\% and 5\% $\mathrm{NaCl}$ ) was selected for identification by $16 \mathrm{~S} \mathrm{r}$ RNA gene sequencing. These identified selected single rhizobacteria were used for seed treatments under salt stress conditions (data not shown here).

\section{Identification of bacterial strain using $16 \mathrm{~S}$ rRNA gene}

The bacterial strain was identified using standard method of $16 \mathrm{~S}$ rRNA gene sequencing. DNA template was prepared by picking individual colony of each strain and amplification of 16S rRNA gene was carried out by PCR. PCR amplification of DNA was done using universal primers: 27F (5'AGAGTTTGATCMTGGCTCAG-3') and 1492R (5'-TACGGYTACCTTGTT ACGAC TT-3'). PCR reaction mixture $(20 \mu \mathrm{L})$ prepared for full-length 16S rRNA gene amplification was initially denatured at $94^{\circ} \mathrm{C}$ for $3 \mathrm{~min}$, followed by 30 cycles consisting of denaturation at $94^{\circ} \mathrm{C}$ for $30 \mathrm{sec}$, primer annealing at $50^{\circ} \mathrm{C}$ for $60 \mathrm{sec}$ followed by $72^{\circ} \mathrm{C}$ for $60 \mathrm{sec}$ and primer extension at $72^{\circ} \mathrm{C}$ for $10 \mathrm{~min}$ in a thermocycler. Unincorporated PCR primers and dNTPs were removed from PCR products using Montage PCR Clean up kit (Millipore).

The PCR product was sequenced using the 27F/1492R primers. Sequencing reactions were performed using a ABI PRISM $^{\circledR}$ BigDyeTM Terminator Cycle Sequencing Kits with AmpliTaq ${ }^{\circledR}$ DNA polymerase (FS enzyme) (Applied Biosystems).

The purified PCR product samples were sequenced using DNA sequencing service of
TRIYAT SCIENTIFIC CO., Nagpur, Maharashtra, India (https://www.indiamart.com/triyatscientific/\#) using universal 16S rRNA gene sequencing primers (27F/1492R). The sequence results were blast through NCBI (https://blast.ncbi.nlm.nih.gov) and sequence of all the related species were retrieved to get the exact nomenclature of the isolates. Phylogenetic analyses were performed using bioinformatics software PhyML 3.0 aLRT and HKY85 as Substitution model. Other software MUSCLE 3.7 was used for sequence alignments (Edgar, 2004) and aligned sequences were cured using the program Gblocks 0.91b (Talavera and Castrresana, 2007).

\section{Statistical analysis}

All data were presented as Mean \pm SEM of three replicates and analyzed using a statistical package, SPSS (Version 16.0). One-way ANOVA (analysis of variance) was employed followed by Duncan's multiple range tests to determine the significant difference among means of the treatment at $P$ $\leq 0.05$.

\section{Results and Discussion}

\section{Phosphorus solubilization efficiency}

Observation related to phosphorus solubilization efficiency was recorded qualitatively, from different isolated rhizobacterial strains at 5, 10 and 15 days after inoculation (Table 1). There was a significant difference observed among isolated rhizobacteria. The maximum solubilization efficiency was recorded in $\mathrm{CH}$ red strain $(348.08 \%)$ at 5 days after inoculation (DAI), followed by BP red (339.05\%) and T1 (309.44\%) strain, but at 10 and 15 DAI, rhizobacteria BP red $(607.78 \%$ and $621.43 \%$ ), showed maximum efficiency 
followed by $\mathrm{CH}$ red $(545.56 \%$ and $548.41 \%)$ and $\mathrm{T} 3$ white $(350.40 \%$ and $375.93 \%$, respectively). Some rhizobacteria did not solubilise tri-calcium phosphate i.e., $\mathrm{CH}$ yellow, $\mathrm{BP}$ white, $\mathrm{CH}$ white and $\mathrm{T} 3$ white.

Phosphate-solubilizing bacteria have ability to solubilize the insoluble forms of the phosphate. The primary mechanism of phosphate solubilization is based on organic acid secretion by microbes because of sugar metabolism. Organisms residing in the rhizosphere utilize sugars from root exudates, metabolize them to produce organic acids (Goswami et al., 2015). These acids released by the micro-organisms act as good chelators of divalent $\mathrm{Ca}^{2+}$ cations accompanying the release of phosphates from insoluble phosphatic compounds. Many of the phosphate-solubilizing microbes lower the $\mathrm{pH}$ of the medium by secretion of organic acids such as acetic, lactic, malic, succinic, tartaric, gluconic, 2-ketogluconic, oxalic and citric acids (Rodríguez and Fraga, 1999, Patel et al., 2015) (Fig. 1).

\section{Potassium solubilization efficiency}

Data presented in Table 1 related to potassium solubilization efficiency were recorded qualitatively from different isolated rhizobacterial strains at 5,10 and 15 DAI. Significant difference was observed among isolated rhizobacteria. There was maximum solubilization efficiency recorded in $\mathrm{C} 1$ (467.22\%) followed by T2 brown (444.44\%) and $\mathrm{CH}$ red $(366.67 \%)$ at $5 \mathrm{DAI}$, but at 10 DAI, observed maximum solubilization efficiency in BP red $(685.35 \%)$ followed by $\mathrm{C} 1 \quad(669.99 \%)$ and $\mathrm{CH}$ yellow (640.0\%). Similarly, at 15 DAI, maximum solubilization efficiency was observed in C1 (702.38\%) followed by in $\mathrm{CH}$ red $(649.21 \%)$ and BP red $(615.0 \%)$. Some rhizobacteria did not solubilise organic potassium i.e., T1, P2, CH white and T3 white. Potassium is also an essential macronutrient for the plants that helps in several metabolic processes to maintain cytosolic ions balance. Several rhizospheric bacteria have capacity to solubilise potassium by releasing organic acids (Sheng 2005; Han, 2006; Badri, 2006; Basak and Biswas, 2010; Singh et al., 2010).

\section{Siderophore production}

Efficiency of siderophore production is presented in Table 2 for evaluation of different isolated rhizobacteria from rhizospheres of brinjal, chilli and tomato plants. There was a significant difference among isolated rhizobacteria. Maximum siderophore production capability was observed in $\mathrm{T} 2$ brown (211.52\%) followed by C1 $(81.30 \%)$ and C2 (76.46\%). These rhizobacteria followed same trend at 10 and 15 DAI. The PGPR assay tests for all rhizobacterial strains produce siderophore that are helpful directly and/or indirectly in plant growth and development.

Siderophore-producing bacteria usually belong to the genus Pseudomonas, where the most studied organisms are Pseudomonas fluorescens and Pseudomonas aeruginosa which release pyochelin and pyoverdine type of siderophores (Haas and Defago, 2005). Rhizosphere bacteria release these compounds to increase their competitive potential, since these substances have an antibiotic activity and increase availability of iron for the plant growth (Glick, 1995). Siderophore-producing rhizobacteria improve plant health at various levels: they improve iron nutrition by forming iron-siderophore complex, inhibit the growth of pathogenic micro-organisms with the release of their antibiotic molecule and inhibit the growth of pathogens by limiting the iron available for the pathogen, generally fungi, which are unable to absorb the iron-siderophore complex (Shen et al., 2013). 
Table.1 Phosphorus and Potassium solubilization efficiency index in different bacterial strains isolated from rhizospheres of Brinjal, Chilli and Tomato plants

\begin{tabular}{|c|c|c|c|c|c|c|}
\hline \multirow{2}{*}{$\begin{array}{l}\text { Parameters } \\
\text { Isolates }\end{array}$} & \multicolumn{3}{|c|}{$\begin{array}{l}\text { Phosphorus Solubilization Efficiency } \\
\text { Index }\end{array}$} & \multicolumn{3}{|c|}{$\begin{array}{c}\text { Potassium Solubilization Efficiency } \\
\text { Index }\end{array}$} \\
\hline & $\begin{array}{l}\text { Inoculation } \\
\text { after } 5 \text { days }\end{array}$ & $\begin{array}{c}\text { Inoculation } \\
\text { after } 10 \\
\text { days }\end{array}$ & $\begin{array}{l}\text { Inoculati } \\
\text { on after } \\
15 \text { days }\end{array}$ & $\begin{array}{l}\text { Inoculation } \\
\text { after } 5 \text { days }\end{array}$ & $\begin{array}{c}\text { Inoculatio } \\
\text { n after } 10 \\
\text { days }\end{array}$ & $\begin{array}{l}\text { Inoculation } \\
\text { after } 15 \text { days }\end{array}$ \\
\hline CH Yellow & $0.00 \pm 0.00^{f}$ & $0.00 \pm 0.00^{\mathrm{g}}$ & $\begin{array}{l}0.00 \pm \\
0.00^{\mathrm{h}}\end{array}$ & $\begin{array}{c}315.00 \pm \\
18.03^{\mathrm{c}}\end{array}$ & $\begin{array}{l}640.00 \pm \\
23.09^{\mathrm{ab}}\end{array}$ & $\begin{array}{l}632.05 \pm \\
16.06^{\mathrm{b}}\end{array}$ \\
\hline $\mathbf{T 1}$ & $\begin{array}{l}309.44 \pm \\
5.80^{\mathrm{b}}\end{array}$ & $\begin{array}{l}221.98 \pm \\
21.42^{\mathrm{d}}\end{array}$ & $\begin{array}{c}208.09 \pm \\
11.81^{\mathrm{e}}\end{array}$ & $0.00 \pm 0.00^{\mathrm{e}}$ & $0.00 \pm$ & $0.00 \pm 0.00^{\mathrm{e}}$ \\
\hline T2 brown & $\begin{array}{c}149.44 \pm \\
12.92^{\mathrm{e}}\end{array}$ & $\begin{array}{l}133.97 \pm \\
3.31^{\mathrm{f}}\end{array}$ & $\begin{array}{c}145.83 \pm \\
10.49^{\mathrm{g}}\end{array}$ & $\begin{array}{c}444.44 \pm \\
18.19^{\mathrm{a}}\end{array}$ & $\begin{array}{c}449.05 \pm \\
12.24^{\mathrm{c}}\end{array}$ & $\begin{array}{l}503.80 \pm \\
30.51^{\mathrm{c}}\end{array}$ \\
\hline $\mathbf{P 2}$ & $0.00 \pm 0.00^{f}$ & $\begin{array}{l}154.85 \pm \\
2.89^{\mathrm{f}}\end{array}$ & $\begin{array}{c}177.38 \pm \\
4.29^{\mathrm{f}}\end{array}$ & $0.00 \pm 0.00^{\mathrm{e}}$ & $\begin{array}{c}0.00 \pm \\
0.00^{\mathrm{e}}\end{array}$ & $0.00 \pm 0.00^{\mathrm{e}}$ \\
\hline BP White & $0.00 \pm 0.00^{f}$ & $0.00 \pm 0.00^{\mathrm{g}}$ & $\begin{array}{c}0.00 \pm \\
0.00^{\mathrm{h}}\end{array}$ & $\begin{array}{r}248.33 \pm \\
16.41^{\mathrm{d}}\end{array}$ & $\begin{array}{c}281.31 \pm \\
6.65^{\mathrm{d}}\end{array}$ & $\begin{array}{c}280.95 \pm \\
19.05^{\mathrm{d}}\end{array}$ \\
\hline $\mathrm{C} 2$ & $\begin{array}{c}168.89 \pm \\
11.60^{\mathrm{de}}\end{array}$ & $\begin{array}{c}176.01 \pm \\
23.19^{\mathrm{ef}}\end{array}$ & $\begin{array}{c}195.05 \pm \\
16.23^{\mathrm{ef}}\end{array}$ & $\begin{array}{c}230.00 \pm \\
25.17^{\mathrm{d}}\end{array}$ & $\begin{array}{c}322.86 \pm \\
4.36^{\mathrm{d}}\end{array}$ & $\begin{array}{c}321.43 \pm \\
10.91^{\mathrm{d}}\end{array}$ \\
\hline C1 & $\begin{array}{c}181.62 \pm \\
2.38^{\mathrm{d}}\end{array}$ & $\begin{array}{c}218.06 \pm \\
26.61^{\mathrm{de}}\end{array}$ & $\begin{array}{c}252.98 \pm \\
14.22^{\mathrm{d}}\end{array}$ & $\begin{array}{c}467.22 \pm \\
4.34^{\mathrm{a}}\end{array}$ & $\begin{array}{c}669.99 \pm \\
12.94^{\mathrm{a}}\end{array}$ & $\begin{array}{c}702.38 \pm \\
23.13^{\mathrm{a}}\end{array}$ \\
\hline CH White & $0.00 \pm 0.00^{f}$ & $0.00 \pm 0.00^{\mathrm{g}}$ & $\begin{array}{c}0.00 \pm \\
0.00^{\mathrm{h}}\end{array}$ & $0.00 \pm 0.00^{\mathrm{e}}$ & $\begin{array}{c}0.00 \pm \\
0.00^{\mathrm{e}}\end{array}$ & $0.00 \pm 0.00^{\mathrm{e}}$ \\
\hline CH Red & $\begin{array}{c}348.08 \pm \\
17.70^{\mathrm{a}}\end{array}$ & $\begin{array}{c}545.56 \pm \\
18.49^{\mathrm{b}}\end{array}$ & $\begin{array}{c}548.41 \pm \\
11.03^{\mathrm{b}}\end{array}$ & $\begin{array}{c}366.67 \pm \\
29.06^{\mathrm{b}}\end{array}$ & $\begin{array}{c}614.02 \pm \\
36.21^{\mathrm{b}}\end{array}$ & $\begin{array}{c}649.21 \pm \\
19.94^{\mathrm{ab}}\end{array}$ \\
\hline BP Red & $\begin{array}{c}339.05 \pm \\
19.54^{\mathrm{a}}\end{array}$ & $\begin{array}{c}607.78 \pm \\
16.81^{\mathrm{a}}\end{array}$ & $\begin{array}{c}621.43 \pm \\
14.87^{\mathrm{a}}\end{array}$ & $\begin{array}{c}350.00 \pm \\
14.43^{b c}\end{array}$ & $\begin{array}{c}685.35 \pm \\
18.37^{\mathrm{a}}\end{array}$ & $\begin{array}{c}615.00 \pm \\
35.47^{\mathrm{b}}\end{array}$ \\
\hline CP Red & $\begin{array}{c}234.04 \pm \\
8.83^{\mathrm{c}}\end{array}$ & $\begin{array}{c}350.40 \pm \\
17.26^{\mathrm{c}}\end{array}$ & $\begin{array}{c}375.93 \pm \\
7.28^{\mathrm{c}}\end{array}$ & $\begin{array}{c}220.00 \pm \\
23.09^{d}\end{array}$ & $\begin{array}{c}420.63 \pm \\
21.58^{c}\end{array}$ & $\begin{array}{c}551.03 \pm \\
14.01^{\mathrm{c}}\end{array}$ \\
\hline T3 White & $0.00 \pm 0.00^{f}$ & $0.00 \pm 0.00^{\mathrm{g}}$ & $\begin{array}{c}0.00 \pm \\
0.00^{\mathrm{h}}\end{array}$ & $0.00 \pm 0.00^{\mathrm{e}}$ & $\begin{array}{c}0.00 \pm \\
0.00^{\mathrm{e}}\end{array}$ & $0.00 \pm 0.00^{\mathrm{e}}$ \\
\hline
\end{tabular}

where, $\mathrm{CH}$ Yellow, T1, T2 brown, P2, BP White, C2, C1, CH White, CH Red, BP Red, CP Red and T3 White are different bacterial strains isolated from rhizosphere of different plants i.e. brinjal, chilli and tomato; Data are in the form of mean \pm SEM, and means followed by the same letters within the columns are not significantly different at $P \leq 0.05$ using Duncan's multiple range test 
Table.2 Production of siderophore, auxin and hydrogen cyanide by different isolated rhizobacteria from brinjal, chilli and tomato plants

\begin{tabular}{|c|c|c|c|c|c|}
\hline \multirow{2}{*}{$\begin{array}{l}\text { Parameters } \\
\text { Isolates }\end{array}$} & \multicolumn{3}{|c|}{ Siderophore Production } & \multirow{2}{*}{$\begin{array}{c}\text { Auxin } \\
\text { Production } \\
\text { Diameter }(\mathrm{cm}) \\
\text { of red halo on } \\
\text { filter paper } \\
\text { after one day }\end{array}$} & \multirow{2}{*}{$\begin{array}{c}\text { HCN } \\
\text { Production } \\
\text { Through } \\
\text { Visualization }\end{array}$} \\
\hline & $\begin{array}{l}\text { Inoculation } \\
\text { after } 5 \text { days }\end{array}$ & $\begin{array}{l}\text { Inoculation } \\
\text { after } 10 \text { days }\end{array}$ & $\begin{array}{l}\text { Inoculation } \\
\text { after } 15 \text { days }\end{array}$ & & \\
\hline CH Yellow & $66.75 \pm 3.42^{b}$ & $42.84 \pm 0.55^{\mathrm{d}}$ & $44.54 \pm 1.02^{\mathrm{g}}$ & $0.80 \pm 0.06^{\mathrm{c}}$ & +++ \\
\hline T1 & $69.17 \pm 4.47^{b}$ & $78.11 \pm 0.40^{b}$ & $78.82 \pm 1.82^{b c}$ & $0.50 \pm 0.06^{\mathrm{e}}$ & - \\
\hline T2 brown & $211.52 \pm 1.92^{\mathrm{a}}$ & $211.81 \pm 8.52^{\mathrm{a}}$ & $200.75 \pm 6.80^{\mathrm{a}}$ & $0.00 \pm 0.00^{\mathrm{g}}$ & - \\
\hline P2 & $15.08 \pm 2.21^{\mathrm{d}}$ & $71.81 \pm 4.14^{\mathrm{b}}$ & $78.87 \pm 5.12^{\mathrm{bc}}$ & $0.00 \pm 0.00^{g}$ & - \\
\hline BP White & $14.91 \pm 3.73^{d}$ & $72.34 \pm 5.42^{b}$ & $83.61 \pm 2.17^{b}$ & $0.00 \pm 0.00^{\mathrm{g}}$ & + \\
\hline $\mathrm{C} 2$ & $76.46 \pm 6.99^{b}$ & $81.20 \pm 0.68^{b}$ & $84.11 \pm 0.90^{b}$ & $0.00 \pm 0.00^{\mathrm{g}}$ & - \\
\hline C1 & $81.30 \pm 4.69^{b}$ & $77.05 \pm 2.60^{b}$ & $83.78 \pm 2.21^{b}$ & $0.48 \pm 0.04^{\mathrm{e}}$ & - \\
\hline CH White & $5.81 \pm 2.91^{d}$ & $57.04 \pm 3.53^{c}$ & $78.99 \pm 1.62^{b c}$ & $0.30 \pm 0.03^{f}$ & - \\
\hline CH Red & $75.27 \pm 9.59^{b}$ & $47.78 \pm 2.28^{\mathrm{cd}}$ & $55.71 \pm 2.38^{\mathrm{ef}}$ & $0.63 \pm 0.06^{\mathrm{d}}$ & ++ \\
\hline BP Red & $47.16 \pm 2.60^{c}$ & $54.66 \pm 2.26^{c}$ & $72.44 \pm 5.32^{\text {cd }}$ & $1.05 \pm 0.08^{b}$ & +++ \\
\hline CP Red & $66.51 \pm 2.74^{b}$ & $58.86 \pm 0.41^{\mathrm{c}}$ & $63.23 \pm 1.81^{\mathrm{de}}$ & $0.75 \pm 0.03^{\mathrm{cd}}$ & ++ \\
\hline T3 White & $44.98 \pm 2.62^{c}$ & $43.47 \pm 2.17^{d}$ & $49.29 \pm 1.22^{\mathrm{fg}}$ & $1.70 \pm 0.06^{\mathrm{a}}$ & + \\
\hline
\end{tabular}

where, Yellow, T1, T2 brown, P2, BP White, C2, C1, CH White, CH Red BP Red CP Red and T3 White are different bacterial strains isolated from rhizosphere of different plants i.e. brinjal, chilli and tpmato, $+++=$ High intensity (break red), $++=$ Medium intensity (break red), $+=$ Low intensity (break red), - = No intensity (break red), Data are in the form of mean \pm SEM, and means followed by the same letters within the columns are not significantly different at $P \leq 0.05$ using Duncan's multiple range test

Table.3 Colony Forming Unit $\left(\mathrm{CFU} \mathrm{mL} \mathrm{mL}^{-1}\right)$ of different bacterial strains isolated from rhizospheres of brinjal, chilli and tomato plants under $2 \%$ and $5 \% \mathrm{NaCl}$ solutions

\begin{tabular}{|c|c|c|c|c|}
\hline \multirow{2}{*}{ Isolates } & \multicolumn{2}{|c|}{$\begin{array}{c}\text { Parameters } \\
\text { Sacterial broth with 2\% NaCl } \\
\text { solution }\end{array}$} & \multicolumn{2}{|c|}{$\begin{array}{c}\text { Bacterial broth with 5\% NaCl } \\
\text { solution }\end{array}$} \\
\cline { 2 - 5 } & $\begin{array}{c}\text { CFU after 7 } \\
\text { days }\end{array}$ & $\begin{array}{c}\text { CFU after 14 } \\
\text { days }\end{array}$ & $\begin{array}{c}\text { CFU after 7 } \\
\text { days }\end{array}$ & $\begin{array}{c}\text { CFU after 14 } \\
\text { days }\end{array}$ \\
\hline CH Yellow & $2.69 \times 10^{9}$ & $5.07 \times 10^{9}$ & $2.74 \times 10^{8}$ & $4.58 \times 10^{7}$ \\
\hline T1 & $3.48 \times 10^{8}$ & $1.53 \times 10^{9}$ & $2.09 \times 10^{8}$ & $1.62 \times 10^{8}$ \\
\hline T2 brown & $3.16 \times 10^{7}$ & $1.35 \times 10^{8}$ & $4.27 \times 10^{6}$ & $1.94 \times 10^{7}$ \\
\hline P2 & $4.29 \times 10^{6}$ & $3.58 \times 10^{7}$ & $3.42 \times 10^{6}$ & $6.20 \times 10^{5}$ \\
\hline BP White & $5.82 \times 10^{8}$ & $1.43 \times 10^{9}$ & $1.68 \times 10^{8}$ & $4.11 \times 10^{8}$ \\
\hline C2 & $4.64 \times 10^{7}$ & $2.16 \times 10^{8}$ & $1.58 \times 10^{7}$ & $7.19 \times 10^{6}$ \\
\hline C1 & $3.48 \times 10^{8}$ & $2.61 \times 10^{9}$ & $1.67 \times 10^{8}$ & $3.25 \times 10^{8}$ \\
\hline CH White & $4.72 \times 10^{10}$ & $6.47 \times 10^{9}$ & $2.43 \times 10^{9}$ & $3.68 \times 10^{9}$ \\
\hline CH Red & $1.61 \times 10^{8}$ & $2.71 \times 10^{8}$ & $4.54 \times 10^{7}$ & $4.72 \times 10^{6}$ \\
\hline BP Red & $4.53 \times 10^{8}$ & $1.22 \times 10^{9}$ & $1.11 \times 10^{8}$ & $6.13 \times 10^{7}$ \\
\hline CP Red & $5.27 \times 10^{9}$ & $1.26 \times 10^{10}$ & $2.61 \times 10^{8}$ & $4.69 \times 10^{7}$ \\
\hline T3 White & $2.63 \times 10^{9}$ & $6.94 \times 10^{8}$ & $4.38 \times 10^{8}$ & $1.25 \times 10^{8}$ \\
\hline
\end{tabular}

where, CH Yellow, T1, T2 brown, P2, BP White, C2, C1, CH White, CH Red, BP Red, CP Red and T3 White are different bacterial strains isolated from rhizosphere of different plants i.e. brinjal, chilli and tomato 
Table.4 Bio-fertilizer activity and efficiency of different bacterial strains isolated from rhizospheres of brinjal, chilli and tomato plants

\begin{tabular}{|c|c|c|c|c|c|}
\hline Isolates & $\begin{array}{c}\text { Phosphate } \\
\text { solubilization }\end{array}$ & $\begin{array}{c}\text { Potassium } \\
\text { solubilization }\end{array}$ & $\begin{array}{c}\text { Siderophore } \\
\text { production }\end{array}$ & $\begin{array}{c}\text { Auxin } \\
\text { production }\end{array}$ & $\begin{array}{c}\text { HCN } \\
\text { Production }\end{array}$ \\
\hline CH Yellow & - & +++ & + & ++ & +++ \\
\hline T1 & + & - & ++ & + & - \\
\hline T2 brown & + & ++ & +++ & - & - \\
\hline P2 & + & - & ++ & - & + \\
\hline BP White & - & + & ++ & - & - \\
\hline C2 & + & + & ++ & + & - \\
\hline C1 & + & +++ & ++ & + & + \\
\hline CH White & - & - & ++ & + & ++ \\
\hline CH Red & +++ & +++ & + & + & \\
\hline BP Red & +++ & +++ & ++ & + & + \\
$\left(\begin{array}{c}\text { Pseudomonas } \\
\text { aeruginosa }\end{array}\right.$ & & & + & + & + \\
\hline CP Red & ++ & + & ++ & + \\
\hline T3 White & - & - & + & + & + \\
\hline
\end{tabular}

where, $\mathrm{CH}$ Yellow, T1, T2 brown, P2, BP White, C2, C1, CH White, CH Red BP Red CP Red and T3 White are different bacterial strains isolated from rhizosphere of different plants i.e. brinjal, chilli and tomato, and $+++=$ High positive response, $++=$ Medium positive response, $+=$ Low positive response and $-=$ Negative response of rhizobacterial isolates for particular PGPR assay

Fig.1 Phosphorus solubilization (1A), Potassium solubilization (1B), Siderophore production (1C), HCN production (1D) and Auxin production (1E) of isolated rhizobacteria (BP Red)

\begin{tabular}{|c|c|}
\hline Phosphorus solubilization by BP Red & \\
\hline Potassium solubilization by BP Red & \\
\hline Siderophore production by BP Red & \\
\hline HCN production by BP Red & \\
\hline Auxin Production by BP Red & Coutrol \\
\hline
\end{tabular}


Fig.2 Phylogenic Tree of isolated rhizobacteria (BP Red- identified as Pseudomonas aeruginosa as per $16 \mathrm{~S}$ rRNA sequencing

Primer details:

27F (5'-AGAGTTTGATCMTGGCTCAG-3')

1492R (5'-TACGGYTACCTTGTTACGACTT-3')

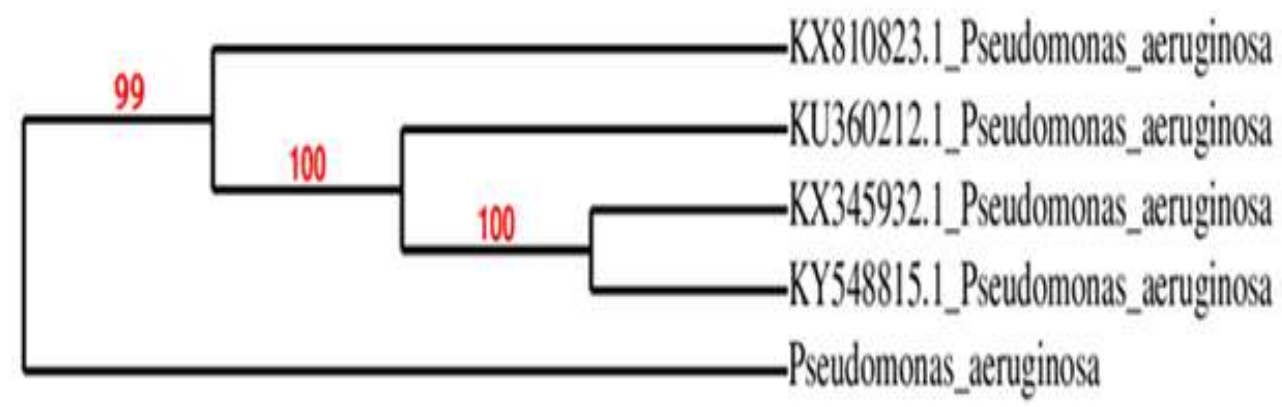

\section{Auxin production}

There was a significant difference observed among isolated rhizobacteria in respect to auxin production, quantitatively (Table 2). Ability of maximum auxin production was recorded in $\mathrm{T} 3$ white $(1.7 \mathrm{~cm})$ followed by $\mathrm{BP}$ red $(1.05 \mathrm{~cm})$ and $\mathrm{CH}$ yellow $(0.8 \mathrm{~cm})$ which developed red halo ( $\mathrm{cm}$ in diameter) on filter paper one day after inoculation. Some rhizobacteria did not synthesise auxin i.e., T2 brown, P2, BP white and C2. Auxins are plant hormones that are essential for plant growth and development.

They have a fundamental role in coordination of many growth and physiological processes in the plant's life. Plant growth promoting bacteria exhibit a variety of characteristics responsible for influencing plant growth including the production of IAA (Fukuhara $e t$ al., 1994, Patten and Glick, 2002). Indole-3acetic acid in rhizobacteria helps loosen plant cell walls, which may facilitate rhizobacteria to absorb various substances secreted by roots (Glick, 2012). Several studies have suggested that elevated auxin levels, including IAA in host plants, are required for nodule formation
(Fukuhara et al., 1994, Mathesius et al., 1998, Pii et al., 2007).

It has been reported that IAA production by bacteria can vary among different species and strains, and it is also influenced by culture condition, growth stage and substrate availability (Mandal et al., 2007).

\section{Hydrogen cyanide (HCN) production}

Hydrogen cyanide produced by the plant growth promoting microorganisms helps protect plants from soil borne pathogens. Intensity of $\mathrm{HCN}$ production was evaluated in different rhizobacteria, isolated from rhizosphere of brinjal, chilli and tomato plants (Table 2). Significant difference was found among isolated rhizobacteria. The maximum intensity of $\mathrm{HCN}$ was found in BP red and $\mathrm{CH}$ yellow followed by $\mathrm{CH}$ red and $\mathrm{CP}$ red. However, some isolated rhizobacteria did not produce $\mathrm{HCN}$ (T1, T2 brown, P2, C2, C1 and $\mathrm{CH}$ white). $\mathrm{HCN}$ produced by several rhizobacteria help plants in their growth and development indirectly by suppression of plant pathogenic populations. Changing the colour of plates from yellow to brown/red 
confirmed HCN production (Castric, 1975; Reetha et al., 2014).

\section{Colony forming unit (CFU) of rhizobacteria under $2 \%$ and $5 \% \mathrm{NaCl}$ solution}

Colony forming unit (CFU) of different isolated rhizobacteria was evaluated for the regeneration abilities of these rhizobacteria under $2 \%$ and $5 \% \mathrm{NaCl}$ solution at 7 and 14 days after inoculation (Table 3 ).

There was a significant reduction in rhizobacterial $\mathrm{CFU}$ with increasing concentration of $\mathrm{NaCl}$. In $2 \% \mathrm{NaCl}$, regeneration abilities in terms of $\mathrm{CFU}$ was observed maximum in $\mathrm{CH}$ white $\left(4.72 \times 10^{10}\right)$ followed by $\mathrm{CP}$ red $\left(5.27 \times 10^{9}\right)$ and $\mathrm{CH}$ yellow $\left(2.69 \times 10^{9}\right)$ at 7 days after inoculation, but after 14 days inoculation, found maximum in $\mathrm{CP}$ red $\left(1.26 \mathrm{x} 10^{10}\right)$ followed by $\mathrm{CH}$ white $\left(6.47 \times 10^{9}\right)$ and $\mathrm{CH}$ yellow $\left(5.07 \times 10^{9}\right)$. CFU under $5 \% \mathrm{NaCl}$ solution was maximum in $\mathrm{CH}$ white $\left(2.43 \times 10^{9}\right)$ followed by $\mathrm{T} 3$ white $(4.38$ $\left.\mathrm{x} 10^{8}\right)$ and $\mathrm{CH}$ yellow $\left(2.74 \times 10^{8}\right)$ at 7 days after inoculation but after 14 days, maximum $\mathrm{CFU}$ was found in $\mathrm{CH}$ white $\left(3.68 \times 10^{9}\right)$ followed by $\mathrm{BP}$ white $\left(4.11 \times 10^{8}\right)$ and $\mathrm{C} 1$ (3.25 x $\left.10^{8}\right) \mathrm{CFU} \mathrm{mL}^{-1}$.

It is concluded that, among isolated rhizospheric strains, BP red performed best in terms of various PGPR assays (Table 4). BP red stain was identified by $16 \mathrm{~S}$ rRNA sequencing and phylogenetic tree showed that the sequence had a high similarity of $99 \%$ with Pseudomonas aeruginosa (KX810823.1) (Fig. 2). Therefore, PGPR strain BP red was confirmed as Pseudomonas aeruginosa based on $16 \mathrm{~S}$ ribosomal RNA sequencing. In the subsequent experiments under stress and ameliorative treatments, henceforth, Pseudomonas aeruginosa has been refered to as rhizobacteria.

\section{Acknowledgement}

Authors thank to the UGC New Delhi for providing fellowship to the first author.

\section{References}

Ahmed, E and Holmstrom, S.J.M. (2014). Siderophores in environmental research: roles and applications. Microb. Biotechnol. 7: 196-208.

Badri, M.A. (2006). Efficiency of K-feldspar combined with organic materials and silicate dissolving bacteria on tomato yield. J. Applied Sci. Res. 2: 11911198.

Basak, B.B and Biswas, D.R. (2010). Coinoculation of potassium solubilizing and nitrogen fixing bacteria on solubilization of waste mica and their effect on growth promotion and nutrient acquisition by a forage crop. Biol. Fertil. Soil. 46: 641-648.

Castric, P.A. (1975). Hydrogen cyanide, a secondary metabolite of Psuedomonas aeruginosa. Can. J. Microbiol. 21: 613-618.

Dobbelaere, A., Vanderleyden, J and Okon, Y. (2003). Plant growth-promoting effects of diazotrophs in the rhizospheres. Crit. Rev. Plant Sci. 2: 107-149.

Edgar, R.C. (2004). MUSCLE: multiple sequence alignment with high accuracy and high throughput. Nucleic Acids Res. 32(5): 1792-1797.

Ehmann, A. (1977). The Van Urk-Salkowski reagent-a sensitive and specific chromogenic reagent for silica gel thin-layer chromatographic detection and identification of indole derivatives. J. Chromatogr. 132: 267276.

Fukuhara, H., Minakawa, Y., Akao, S and Minamisawa, K. (1994). The 
involvement of indole-3 acetic acid produced by Bradyrhizobium elkanii in nodule formation. Plant Cell Physiol. 35: 1261-1265.

Glick, B. R. (2014). Bacteria with ACC deaminase can promote plant growth and help to feed the world. Microbiol. Res. 169: 30-39.

Glick, B.R. (1995). The enhancement of plant growth by free living bacteria. Can. J. Microbiol. 41: 109-117.

Glick, B.R. (2012). Plant growth-promoting bacteria: Mechanisms and applications. Scientifica 2012:1-15.

Goswami, D., Patel, K., Parmar, S., Vaghela, H., Muley, N., Dhandhukia, P and Thakker, J. N. (2015). Elucidating multifaceted urease producing marine Pseudomonas aeruginosa $\mathrm{BG}$ as a cogent PGPR and bio-control agent. Plant Growth Regul. 75(1): 256-263.

Govindasamy, V., Senthilkumar, M., Magheshwaran, V., Kumar, U., Bose, P., Sharma, V., and Annapurna, K. (2011). Bacillus and Paenibacillus spp.: Potential PGPR for sustainable agriculture. In D. K. Maheshwari (Ed.), Plant growth and health promoting bacteria pp. 333-364. Berlin: Springer-Verlag.

Haas, D and Défago, G. (2005). Biological control of soil-borne pathogens by fluorescent Pseudomonads. Nat. Rev. Microbiol. 3: 307-319.

Han, H.S., Supanjani and Lee, K. D. (2006). Effect of co-inoculation with phosphate and potassium solubilizing bacteria on mineral uptake and growth of pepper and cucumber. Plant Soil and Environ. 52: 130-136.

Hayat, R., Ali, S., Amara, U., Khalid, R and Ahmed, I. (2010). Soil beneficial bacteria and their role in plant growth promotion: a review. Ann. Microbiol. 60: 579-598.

Hoitink, H.A.J., Madden, L.V and Dorrance,
A.E. (2006). Systemic resistance induced by Trichoderma spp.: interactions between the host, the pathogen, the biocontrol agent, and soil organic matter quality. Phytopathol. 96: 186-189.

Hu, X., Chen, J and Guo, J. (2006). Two phosphate- and potassium-solubilizing bacteria isolated from Tianmu Mountain, Zhejiang, China. World J. Microbiol. Biotechnol. 22: 983-990.

Jha, C. K., Patel, D., Rajendran, N and Saraf, M. (2010). Combinatorial assessment on dominance and informative diversity of PGPR from rhizosphere of Jatropha curcas L. J. Basic Microbiol. 50:211-217.

Liu, D., Lian, B and Dong, H. (2012). Isolation of Paenibacillus sp. and assessment of its potential for enhancing mineral weathering. Geomicrobiol. J. 29: 413-421.

Mandal, S.M., Mondal, K.C., Dey, S and Pati, B.R. (2007). Optimization of cultural and nutritional conditions for indole3 -acetic acid (IAA) production by a Rhizobium sp. isolated from root nodules of Vigna mungo (L.) Hepper. Res. J. Microbiol. 2: 239-246.

Mathesius, U., Schlaman, H.R., Spaink, H.P., Of Sautter, C., Rolfe, B,G., et al., (1998). Auxin transport inhibition precedes root nodule formation in white clover roots and is regulated by flavonoids and derivatives of chitin oligosaccharides. Plant J. 14: 23-34.

Mathys, J., De Cremer, K., Timmermans, P., Van Kerckhove, S., Lievens, B., Vanhaecke, M., Cammue, B.P and De Coninck, B. (2012). Genome-wide characterization of ISR induced in Arabidopsis thaliana by Trichoderma hamatum T382 against Botrytis cinerea infection. Front. Plant Sci. 3(108): 1-25.

Mehta, S and Nautiyal, C. S. (2001). An 
efficient method for qualitative screening of phosphate-solubilizing bacteria. Curr. Microbiol. 43: 51-56.

Mishra, M., Kumar, U., Mishra, P.K and Prakash, V. (2010). Efficiency of plant growth promoting rhizobacteria for the enhancement of Cicer arietinum $\mathrm{L}$. growth and germination under salinity. Adv. Biol. Res. 4: 92-96.

Park, J., Bolan, N., Megharaj, M and Naidu, R. (2010). Isolation of PhosphateSolubilizing Bacteria and their effects characterization of on Lead Immobilization. Pedologist. 53: 67-75.

Patel, K., Goswami, D., Dhandhukia, P and Thakker, J. (2015). Techniques to study microbial phytohormones. pp. 1-27. In D. K. Maheshwari (Ed.), Bacterial metabolites in sustainable agroecosystem, Springer International.

Patten, C.L and Glick, B.R. (2002). Role of Pseudomonas putida indoleacetic acid in development of the host plant root system. Appl. Environ. Microbiol. 68: 3795- 3801.

Pii, Y., Crimi, M., Cremonese, G., Spena, A and Pandolfini, T. (2007). Auxin and nitric oxide control indeterminate nodule formation. BMC Plant Biol. 7: $1-9$.

Reetha, A.K., Pavani, S.L and Mohan, S. (2014). Hydrogen Cyanide Production Ability by bacterial antagonist and their Antibiotics Inhibition Potential on Macrophomina phaseolina (Tassi.) Goid. Int. J. Curr. Microbiol. App. Sci. 3(5): 172-178.

Rodríguez, H and Fraga, R. (1999). Phosphate solubilizing bacteria and their role in plant growth promotion. Biotechnol. Adv. 17: 319-339.

Schwyn, B and Neilands, J.B. (1987). Universal chemical assay for the detection and determination of siderophores. Anal. Biochem. 160: 47-56.
Shen, X., Hu, H., Peng, H., Wang, W and Zhang, X. (2013). Comparative genomic analysis of four representative plant growth-promoting rhizobacteria in Pseudomonas. BMC Genomics. 14:271-297.

Sheng, X. F. (2005). Growth promotion and increased potassium uptake of cotton and rape by a potassium releasing strain of Bacillus edaphicus. Soil Biol. and Biochemi. 37: 1918-1922.

Shoresh, M., Harman, G.E and Mastouri, F. (2010). Induced systemic resistance and plant responses to fungal biocontrol agents. Annu. Rev. Phytopathol. 48: 21-43.

Singh, A.K and Dwivedi, P. (2018a). Modulation of salt stress induced responses in pea (Pisum sativum L.) through salicylic acid and Trichoderma application. Int. J. Curr. Microbiol. App. Sci. 7(4): 3173-3185.

Singh, A.K and Dwivedi, P. (2018b). Salicylic acid and Trichoderma ameliorate salt stress responses in pea (Pisum sativum L.). Int. J. Agric. Environ. Biotechnol. 11(2):387-395.

Singh, B.N., Singh, A., Singh, G.S and Dwivedi, P. (2015). Potential role of Trichoderma asperellum T42 strain in growth of pea plant for sustainable agriculture. J. Pure Applied Microbiol. 9(2): 1069-1074.

Singh, G., Biswas, D.R and Marwah, T.S. (2010). Mobilization of potassium from waste mica by plant growth promoting rhizobacteria and its assimilation by maize (Zea mays) and wheat (Triticum aestivum L.). J. Plant Nutri. 33: 1236-1251.

Spaepen, S., Versees, W., Gocke, D., Pohl, M., Steyaert, J and Vanderleyden, J. (2007). Characterization of phenylpyruvate decarboxylase, involved in auxin production of Azospirillum brasilense. J. Bacteriol., 
189: 7626-7633.

Talavera, G and Castresana, J. (2007). Improvement of phylogenies after removing divergent and ambiguously aligned blocks from protein sequence alignments. Systematic Biol. 56: 564577.

Tallapragada, P and Seshachala, U. (2010). Phosphate-Solubilizing microbes and their occurrence in the rhizospheres of Piper betel. Biol. 362012: 25-35.
Vessey, J. K. (2003). Plant growth promoting rhizobacteria as biofertilizers. Plant and Soil, 255: 571-586.

Yousefi, A.A., Khavazi, K., Moezi, A.A., Rejali, F and Nadian, H.A. (2011). Phosphate Solubilizing Bacteria and Arbuscular Mycorrhiza Fungi Impacts on Inorganic Phosphorus Fractions and Wheat Growth. World Applied Sci. J. 15 (9): 1310-1318.

\section{How to cite this article:}

Ajay Kumar Singh and Padmanabh Dwivedi. 2019. Identification of Plant Growth Promoting Rhizobacteria as Biofertilizer for Salt Stress Environment. Int.J.Curr.Microbiol.App.Sci. 8(01): 2633-2645. doi: https://doi.org/10.20546/ijcmas.2019.801.277 